\title{
Nonparametric Tests for Data in Randomised Blocks with Ordered Alternatives
}

\author{
J.C.W. RAYNER \\ john_rayner@uow.edu.au \\ School of Mathematics and Applied Statistics, University of Wollongong, Northfields Avenue, \\ NSW 2522, Australia \\ D.J. BEST \\ john.best@cmis.csiro.au \\ CSIRO Mathematical and Information Sciences, PO Box 52, North Ryde, NSW 1670, Australia
}

\begin{abstract}
For randomized block designs, nonparametric treatment comparisons are usually made using the Friedman test for complete designs, and the Durbin test for incomplete designs; see, for example, Conover (1998). This permits assessment of only the mean rankings. Such comparisons are here extended to permit assessments of bivariate effects such as the linear by linear effect and the quadratic by linear, or umbrella effect.
\end{abstract}

Keywords: Balanced incomplete blocks, Friedman's test, Orthonormal polynomials.

\section{Introduction}

Boos (1986) and Nair (1986) gave nonparametric methods for comparing K independent samples for the cases of uncategorised and categorised data respectively. The statistics they gave looked at dispersion effects as well as the usual location effects. Best (1994) noted that these statistics for location and dispersion effects can be obtained via a partition of chi-squared approach, while Best (1993) gave examples showing that the same approach is also useful for randomised block or $\mathrm{K}$-related samples ranked data. In this note we further extend the partition of chi-squared approach to the randomised blocks situation where the products or treatments being compared have an a priori ordering associated with them.

In the $\mathrm{K}$ independent samples case it is known that a monotonic a priori ordering of the samples can be tested using a Jonckheree (1954) statistic and that umbrella alternatives can be tested as in Mack and Wolfe (1981). Best and Rayner (1996) and Rayner and Best (1996a) discussed a Spearman statistic which can also be used to test an a priori monotonic ordering in the $\mathrm{K}$ independent samples situation. In addition Best and Rayner (1996) gave an umbrella test statistic for the $\mathrm{K}$ independent samples situation. In the randomised block case an a priori monotonic ordering can be tested using the well known Page (1963) statistic. Here we introduce a statistic for an umbrella alternative for both complete and incomplete randomised blocks. We also consider the cases of tied data when testing for an ordered alternative. 


\section{Lancaster's Partition}

Suppose we have a K by K two-way table of counts $N_{i j}, i, j=1, \cdots, K$, where both row and column categories are ordered, having associated scores $i$ and $j$ respectively. The usual chi-squared test of homogeneity or independence is

$$
X_{P}^{2}=\sum_{i=1}^{K} \sum_{j=1}^{K} \frac{\left(N_{i j}-E_{i j}\right)^{2}}{E_{i j}}
$$

in which, if $\sum_{i=1}^{K} \sum_{j=1}^{K} N_{i j}=n$,

$$
E_{i j}=\left[\sum_{i=1}^{K} N_{i j}\right]\left[\sum_{j=1}^{K} N_{i j}\right] / n, \quad i, j=1, \cdots, K .
$$

Denote the marginal column probabilities by $\left\{p_{j}\right\}$. For $p_{j}=\frac{1}{K}$ for $j=1, \cdots, K$, we take $\left\{g_{\ell}(j)\right\}$ to be a set of polynomials orthonormal on the discrete uniform distribution. We take $g_{K}(j)=1$ for $j=1, \cdots, K$ and hence, explicitly,

$$
\begin{gathered}
g_{1}(j)=\left(j-\frac{K+1}{2}\right) \sqrt{\frac{12}{K^{2}-1}} \text { and } \\
g_{2}(j)=\left\{\left(j-\frac{K+1}{2}\right)^{2}-\frac{K^{2}-1}{2}\right\} \sqrt{\frac{180}{\left(K^{2}-1\right)\left(K^{2}-4\right)}} .
\end{gathered}
$$

Higher order $g_{\ell}(j)$ may be found via recurrence as in Emerson (1968). Corresponding to the rows we have $\left\{g_{m}(j)\right\}$, again orthonormal on the same discrete uniform distribution. Now take

$$
U_{\ell m}=\sum_{i=1}^{K} \sum_{j=1}^{K} N_{i j} g_{\ell}(i) g_{m}(j) / \sqrt{n} \text { for } \ell, m=1, \cdots, K-1 .
$$

Lancaster (1953) showed that

$$
X_{P}^{2}=\sum_{\ell=1}^{K-1} \sum_{m=1}^{K-1} U_{\ell m}^{2}
$$

and suggested that the $U_{\ell m}$ are asymptotically mutually independent each with the standard normal distribution.

\section{Anderson's Statistic}

Anderson (1959) proposed a nonparametric test for the analysis of randomised block data. Suppose $n$ subjects rank $K$ objects, that no ties are allowed, and that $N_{i j}$ is 
the number of times treatment $i$ receives rank $j$. It is easily shown that for all $i$ and $j, N_{i 1}+\cdots+N_{i K}=N_{1 j}+\cdots+N_{K j}=n$, and that $\sum_{i=1}^{K} \sum_{j=1}^{K} N_{i j}=n K$. Now

$$
A=\frac{K}{n} \sum_{i=1}^{K} \sum_{j=1}^{K}\left(N_{i j}-\frac{n}{K}\right)^{2},
$$

which is of the form of the usual $X_{P}^{2}$ statistic for homogeneity or independence. If we put, as in the previous section,

$$
A=\sum_{\ell=1}^{K-1} \sum_{m=1}^{K-1} U_{\ell m}^{2}
$$

where $n K$ is now used for $n$ in the definition of $U_{\ell m}$, we find $U_{\ell m}$ is no longer well approximated by the standard normal distribution and A is no longer well approximated by the chi-squared distribution. As Anderson (1959, p.582) pointed out, this "is not the usual problem of a contingency table with fixed border totals, because repeated sampling is not a random rearrangement of $(K n)$ items, subject to border restrictions". Both Anderson (1959) and Schach (1979) showed that $\left(\frac{K-1}{K}\right) A$ asymptotically has the $\chi_{(K-1)^{2}}^{2}$ distribution. It is shown in the Appendix that the $U_{\ell m} \sqrt{\frac{K-1}{K}}$ are asymptotically mutually independent, each asymptotically having the standard normal distribution. This not only verifies the Anderson and Schach results, but permits closer scrutiny of the data through the use of the components $U_{\ell m} \sqrt{\frac{K-1}{K}}$.

\section{Page and Umbrella Tests}

(i) Page Test Page's test is used to test for an a priori monotonic ordered alternative. To define Page's test statistic when there are no ties, first suppose observations are ranked separately within each block, and that $r_{i j}$ is the rank of the $i$ th treatment in block $j$, where $i=1, \cdots, K$ and $j=1, \cdots, n$. With $R_{i}=$ $r_{i 1}+\cdots+r_{i n}, i=1, \cdots, K$, the Page test statistic is

$$
L=\sum_{i=1}^{K} i R_{i} .
$$

As $n \rightarrow \infty, L$ approaches normality with mean and variance given by

$$
\mu_{L}=n K(K+1)^{2} / 4 \text { and } \sigma_{L}^{2}=n K^{2}(K-1)(K+1)^{2} / 144
$$

respectively. It is shown in the Appendix that

$$
U_{11} \sqrt{\frac{K-1}{K}}=\frac{L-\mu_{L}}{\sigma_{L}}=Z_{L} \text { say. }
$$


Note that we do not claim that $U_{11} \sqrt{\frac{K-1}{K}}$ provides a new test statistic; only that Page's test can be derived, like many common nonparametric tests, as a component of $X_{P}^{2}$.

(ii) Umbrella Test We suggest that when rows are treatments and columns are ranks, $U_{21} \sqrt{\frac{K-1}{K}}$ be used as an umbrella test statistic. This statistic is analogous to the $K$ independent samples statistic which Best and Rayner (1996) showed gives a value similar to the Mack and Wolfe (1981) umbrella test statistic.

Lemonade Example Consider the set of ranked data shown in Table 1 where there is an a priori ordering of the products A, B, C, D and E.

The Friedman test gives a Monte Carlo p-value of 0.55 and the Page test gives a Monte Carlo p-value of 0.63 . These p-values were obtained using the StatXact (1995) package. Thus two of the standard analyses for the Table 1 data do not indicate significance at the usual 0.05 level. The data in Table 1 can be written as a 5 by 5 table of counts as in Table 2 .

Table 1 . Five products ranked by 10 subjects

\begin{tabular}{cccccc}
\hline & \multicolumn{5}{c}{ Product } \\
Subject & A & B & C & D & E \\
\hline 1 & 5 & 2 & 1 & 3 & 4 \\
2 & 3 & 5 & 2 & 1 & 4 \\
3 & 4 & 3 & 2 & 5 & 1 \\
4 & 5 & 2 & 1 & 3 & 4 \\
5 & 3 & 5 & 2 & 1 & 4 \\
6 & 4 & 3 & 2 & 5 & 1 \\
7 & 5 & 2 & 1 & 3 & 4 \\
8 & 3 & 5 & 2 & 1 & 4 \\
9 & 1 & 4 & 2 & 5 & 3 \\
10 & 3 & 1 & 2 & 4 & 5 \\
\hline
\end{tabular}

Table 2. $\left\{N_{i j}\right\}$ for Table 1 data

\begin{tabular}{cccccc}
\hline & \multicolumn{5}{c}{ Rank } \\
\hline Products & 1 & 2 & 3 & 4 & 5 \\
\hline A & 1 & 0 & 4 & 2 & 3 \\
B & 1 & 3 & 2 & 1 & 3 \\
C & 3 & 7 & 0 & 0 & 0 \\
D & 3 & 0 & 3 & 1 & 3 \\
E & 2 & 0 & 1 & 6 & 1 \\
\hline
\end{tabular}


From this table we can calculate $U_{11}$ and $U_{21}$. The statistic $U_{11} \sqrt{\frac{K-1}{K}}$ gives a standardised version of the Page statistic, $L$. We find $L=445, \mu_{L}=450, \sigma_{L}^{2}=250$ and $z_{L}=\left(L-\mu_{L}\right) / \sigma_{L}=-0.3163$, while $U_{11} \sqrt{\frac{K-1}{K}}=-0.3163$ also. Further $U_{21} \sqrt{\frac{K-1}{K}}=2.2984$ which is significantly large. Thus as we go from $\mathrm{A}$ to $\mathrm{E}$ there are more 3,4 and 5 rankings for $\mathrm{A}$ and $\mathrm{E}$ and more 1 and 2 rankings for $\mathrm{C}$. There is a significant quadratic by linear or "umbrella" effect. This sort of effect has been previously demonstrated in a number of application areas. For the Table 1 data the products $\mathrm{A}$ to $\mathrm{E}$ might be linearly increasing sugar levels in a lemonade drink where the "just right" level of sugar is that in lemonade drink C. Also notice that $U_{21}^{2} / A$ is only $16 \%$ and so there may be other important components of $\mathrm{A}$. We will not pursue this here.

In passing note that the standards Australia document AS2542.2.6 (1995, Table B2-2) and the ISO document ISO8587 (1988, Table A.1) both give a taste-test example where a Page test was applied but where an umbrella test would also have been useful.

\section{Page and Umbrella Tests for Balanced Incomplete Block designs} The partition of $X^{2}$ approach is also useful in that $U_{11}$ and $U_{21}$ (or other $U_{\ell m}$ ) can similarly be calculated for ranked data from balanced incomplete block (BIB) designs. Schach (1979, p.545) also refers to a Page test for BIB data. Rayner and Best (1996b) gave an example of a BIB application where it was useful to calculate $U_{11}$. In order to specify the analogous tests, we use the description and notation from Conover (1998, section 5.9). Each of the $b$ blocks contains $k$ experimental units, each of the $t$ treatments appears in $r$ blocks, and every treatment appears with every other treatment precisely $\lambda$ times. Necessarily $k<t, r<b, b k=r t$, and $\lambda(t-1)=r(k-1)$. The data may be presented in a $t$ by $k$ table of counts $\left\{N_{i j}\right\}$. Put

$$
L=\sum_{i=1}^{t} \sum_{j=1}^{k} i j N_{i j}, \quad \mu_{L}=\frac{r t(k+1)(t+1)}{4} \text { and } \sigma_{L}^{2}=\frac{r t^{2}\left(k^{2}-1\right)(t+1)}{144} .
$$

Then $Z_{L}=\frac{L-\mu_{L}}{\sigma_{L}}$ is a Page statistic that may be compared with the standard normal distribution to assess an a priori monotonic ordering. To assess an $(\ell, m)$ th effect, and in particular a $(2,1)$ umbrella effect, we recommend the test statistics

$$
U_{\ell m}=\sqrt{\frac{t-1}{b k t}} \sum_{i=1}^{t} \sum_{j=1}^{k} N_{i j} g_{\ell}(i) h_{m}(j)
$$

where $\left\{h_{m}(j)\right\}$ is a set of polynomials orthonormal on the discrete uniform with $k$ values whereas $\left\{g_{\ell}(i)\right\}$ is orthonormal on the discrete uniform taking $t$ values. Derivations are parallel to the complete block case and will be omitted here. As in the complete blocks case it can be shown that $Z_{L}$ and $U_{11}$ are the same except for a constant. 
Dried Egg Example In taste-testing there is evidence to suggest that tasting more than four or five samples at one sitting results in reduced acuity arising from confusion or fatigue associated with making a large number of comparative judgements and/or with taste-bud saturation. For this reason incomplete block designs are employed. Rayner and Best (1996b) quote results from a taste-test on ten dried egg samples, $A, B, \ldots, J$, where the design used was a balanced incomplete block design. Table 3 gives the design for 15 sittings and the corresponding mean scores for "off-flavour" from seven judges. If these mean scores are ranked 1, 2, 3, 4 and counts are made of how many times each sample received each rank, then Table 4 results.

Table 3. Balanced incomplete block design and off-flavour scores for dried egg taste-test

\begin{tabular}{ccccccccc}
\hline Sitting & \multicolumn{3}{c}{ Samples } & \multicolumn{4}{c}{ Scores } \\
\hline 1 & A & B & D & E & 9.7 & 8.7 & 5.4 & 5.0 \\
2 & B & C & F & J & 9.6 & 8.8 & 5.6 & 3.6 \\
3 & B & D & F & G & 9.0 & 7.3 & 3.8 & 4.3 \\
4 & A & C & E & G & 9.3 & 8.7 & 6.8 & 3.8 \\
5 & A & D & H & J & 10.0 & 7.5 & 4.2 & 2.8 \\
6 & B & G & H & I & 9.6 & 5.1 & 4.6 & 3.6 \\
7 & B & E & H & J & 9.8 & 7.4 & 4.4 & 3.8 \\
8 & E & G & I & J & 9.4 & 6.3 & 5.1 & 2.0 \\
9 & A & B & C & I & 9.4 & 9.3 & 8.2 & 3.3 \\
10 & D & E & F & I & 8.7 & 9.0 & 6.0 & 3.3 \\
11 & A & F & G & J & 9.7 & 6.7 & 6.6 & 2.8 \\
12 & C & D & I & J & 9.3 & 8.1 & 3.7 & 2.6 \\
13 & A & F & H & I & 9.8 & 7.3 & 5.4 & 4.0 \\
14 & C & D & G & H & 9.0 & 8.3 & 4.8 & 3.8 \\
15 & C & E & F & H & 9.3 & 8.3 & 6.3 & 3.8 \\
\hline
\end{tabular}

Suppose a priori product A is ranked 1, product B is ranked 2, and so on. Then, for these data, $L=988.0, \mu_{L}=825.0$ and $\sigma_{L}^{2}=687.5$, giving $Z_{L}=6.22$ with one-sided p-value 0.000 . Clearly there is a significant ordering of the treatments $A$, $B, \ldots, J$. 
Table 4. Counts of ranks for Table 3 data

\begin{tabular}{ccccc}
\hline & \multicolumn{5}{c}{ Rank } \\
Product & 1 & 2 & 3 & 4 \\
\hline A & 6 & 0 & 0 & 0 \\
B & 4 & 2 & 0 & 0 \\
C & 3 & 2 & 1 & 0 \\
D & 0 & 5 & 1 & 0 \\
E & 2 & 2 & 1 & 1 \\
F & 0 & 2 & 3 & 1 \\
G & 0 & 2 & 3 & 1 \\
H & 0 & 0 & 4 & 2 \\
I & 0 & 0 & 2 & 4 \\
J & 0 & 0 & 0 & 6 \\
\hline
\end{tabular}

6. Tied data If tied rankings are permitted there are various ways in which we could proceed. We will illustrate with reference to Page's test. With tied ranks it is still possible to construct a table of counts $\left\{N_{i j}\right\}$ where, as before, $i=1,2, \cdots, K$ but where now the number of columns depends on which mid-ranks occur. Schach (1979, p.549) noted that the Anderson-type statistic calculated on these counts for the tied data no longer has an asymptotic $\chi^{2}$ distribution. Further, text books and computer software provide different approaches to calculating Page's test. We illustrate by considering an example.

Example Daniel (1990, p.281) gave an example where there are three ordered conditions and 12 subjects with the ranks within subjects as shown in Table 5.

Table 5. Ranks within subjects

\begin{tabular}{cccc}
\hline & \multicolumn{3}{c}{ Conditions } \\
Subject & 1 & 2 & 3 \\
\hline 1 & 2 & 2 & 2 \\
2 & 1 & 3 & 2 \\
3 & 1.5 & 1.5 & 3 \\
4 & 1.5 & 1.5 & 3 \\
5 & 2 & 3 & 1 \\
6 & 2 & 2 & 2 \\
7 & 2.5 & 1 & 2.5 \\
8 & 2.5 & 1 & 2.5 \\
9 & 1 & 2.5 & 2.5 \\
10 & 2 & 2 & 2 \\
11 & 2.5 & 1 & 2.5 \\
12 & 2 & 2 & 2 \\
\hline
\end{tabular}


For these data $L=148.5$. Daniel (1990) stated that $p>0.05$, but he used critical values based on the assumption of no ties. Accounting for ties can make considerable differences in p-values. For example, if we use the approximate standard normal statistic $Z_{L}$ as Daniel did, we find $z_{L}=0.919$ for the Table 5 data, with p-value 0.18. The IMSL (1989) package also gives this result. Conover (1998, p.381) uses the same approximations as Daniel (1990).

A different value of $z_{L}, 1.125$, can be obtained if the four nondiscriminating rows in Table 5 (due to subjects 1, 6, 10 and 12) are omitted. StatXact (1995) gives this value of $Z_{L}$. Pirie (1985) gave a formula for $\sigma_{L}^{2}$ that accounts for ties. If this is used then $z_{L}=1.248$ - a third value! The p-value for the Pirie statistic is 0.11 .

Yet another nonparametric approach to the analysis of these data is to use a Cochran-Mantel-Haenszel (CMH) statistic, the so-called correlation CMH statistic. This is applied with each judge being a stratum, using midranks as scores, and having product totals within each stratum all equal to unity. For the present data the value of the correlation $\mathrm{CMH}$ is equal to the square of the Pirie $Z_{L}$ value. Details of the correlation CMH statistic are given, for example, in Landis et al. (1979).

A permutation test on the Table 5 data is another nonparametric approach, and gives, for the present data, a p-value of 0.14 : close to the Pirie and correlation CMH p-value. Further work needs to be done to confirm that the Pirie $Z_{L}$ (or correlation $\mathrm{CMH}$ statistic) is a preferred approach.

\section{Conclusion}

Nonparametric tests for both complete and incomplete randomised blocks when the treatments have an a priori ordering have been discussed. A partition of chisquared technique was introduced, and this leads to a new test for a quadratic by linear or "umbrella" effect. The case where ranks are tied was considered. We give a proof that the components of chi-squared are asymptotically independent and asymptotically standard normal.

\section{References}

1. Anderson, R.L. Use of contingency tables in consumer preference studies. Biometrics, 15 , $582-590,1959$.

2. AS2542.2.6. Sensory Analysis of Foods - Specific Methods - Ranking. North Sydney: Standards Australia 1995.

3. Best, D.J. Extended analysis for ranked data. Australian Journal of Statistics, 35, 257-262, 1993.

4. Best, D.J. Nonparametric comparison of histograms. Biometrics, 50, 538-541, 1994.

5. Best, D.J. And Rayner, J.C.W. Nonparametric analysis for doubly ordered two-way contingency tables. Biometrics, 52, 1153-1156, 1996.

6. Boos, D. Comparing k populations with linear rank statistics. Journal of the American Statistical Association, 81, 1018-1025, 1986.

7. Conover, W.J. Practical Nonparametric Statistics (3rd ed.) New York: Wiley, 1998.

8. Daniel, W. Applied Nonparametric Statistics (2nd ed.) Boston: PWS Kent, 1990.

9. Emerson, P.L. Numerical construction of orthogonal polynomials from a general recurrence formula. Biometrics 24, 695-701, 1968. 
10. IMSL Library Users Manual Version 1.1 for PCs. Vol 1. Houston: IMSL, 1989.

11. ISO8587.2 Sensory Analysis - Methodology - Ranking. Paris: International Organization for Standardization, 1988.

12. Jonckheree, G.R. A distribution-free k-sample test against ordered alternatives. Biometrika, 41, 133-145, 1954.

13. Lancaster, H.O. A reconciliation of $\chi^{2}$ from metrical and enumerative aspects. Sankhya $13,1-10,1953$.

14. Landis, J.R., Cooper, M.M., Kennedy, T. and Koch, G.G. A computer program for testing partial association in three-way contingency tables (PARCAT). Computer Programs in Biomedicine, 9, 223-246, 1979 .

15. Mack, G.A. and Wolfe, D.A. K-sample rank tests for umbrella alternatives. Journal of American Statistics Association, 76, 175-181, 1981.

16. Nair, V.N. Testing in industrial experiments with ordered categorical data. Technometrics, 28, 283-311, 1986.

17. Page, E.B. Ordered hypotheses for multiple treatments: a significance test for linear ranks. Journal of the American Statistical Association, 58, 216-230, 1963.

18. Pirie, W.R. Page test for ordered alternatives. Encyclopedia of Statistical Sciences. Vol 6 (editors Kotz, S. and Johnson, N.L.), 553-555. New York: Wiley, 1985.

19. Rayner, J.C.W. and Best, D.J. Smooth extensions of Pearson's product moment correlation and Spearman's rho. Statistics and Probability Letters, 30(2), 171-177, 1996a.

20. Rayner, J.C.W. and Best, D.J. Extensions to some important nonparametric tests. In Proceedings of the A.C. Aitken Centenary Conference, Dunedin 1995. (Kavalieris, L. et al. ed.), pp. 257-266. Dunedin: University of Otago Press, 1996b.

21. Schach, S. An alternative to the Friedman test with certain optimality properties. Annals of Statistics, 7, 537-550, 1979.

22. StatXact Statistical Software for Exact Nonparametric Inference. CYTEL Software Corporation, Cambridge MA, 1995.

\section{Appendix}

Distribution of $(K-1) A / K$ and its Components Anderson (1959) noted that

$$
X_{i j}=\left(N_{i j}-n / K\right) \sqrt{n(K-1)}, \text { with } i, j=1, \cdots, K,
$$

are individually asymptotically normally distributed, where $n$ is the number of subjects as in section 3 , and not $\Sigma \Sigma N_{i j}$ as in section 2. Writing

$$
X=\left(X_{11}, \cdots, X_{1 K}, X_{21}, \cdots, X_{2 K}, \cdots, X_{K 1}, \cdots, X_{K K}\right)^{T},
$$

it follows that

$$
[K /(K-1)] A=X^{T} X,
$$

and that $X$ is asymptotically $K^{2}$-variate normal with zero mean and covariance matrix $\Sigma$ say, written $N_{K^{2}}(0, \Sigma)$. This result is implicit in Schach $(1979$, Theorem 2.1). Write $I_{r}$ for the $r$ by $r$ identity matrix, $1_{r}$ for the $r$ by 1 vector of ones and $0_{r}$ for the $r$ by $r$ matrix with every element zero. If

$$
R=[K /(K-1)] I_{K}-[1 /(K-1)] 1_{K} 1_{K}^{T}
$$

then $\Sigma$ is the direct product of $R$ with itself, 


$$
\Sigma=R \otimes R
$$

It is routine to find that the latent roots of $\Sigma$ are $[K /(K-1)]^{2}=d$ say, $(K-1)^{2}$ times, and zero $(2 K-1)$ times. Suppose $H$ is orthogonal and diagonalises $\Sigma$. We then have

$$
H^{T} \Sigma H=d\left(I_{(K-1)^{2}} \oplus 0_{(2 K-1)}\right),
$$

where $\oplus$ means direct sum. Define $Y=[(K-1) / K] H^{T} X$. Then

$$
X^{T} X=d Y^{T} Y
$$

in which $Y$ is asymptotically $N_{K^{2}}\left(0,\left(I_{(K-1)^{2}} \oplus 0_{(2 K-1)}\right)\right)$. It follows that the elements of $Y$ are asymptotically standard normal, and that $(K-1) A / K=X^{T} X / d=$ $Y^{T} Y$ asymptotically has the $\chi_{(K-1)^{2}}^{2}$ distribution, as Anderson (1959) showed, albeit by a different approach.

To identify the components of $(K-1) A / K$ we now define a possible matrix $H$. First suppose that $\left\{g_{r}(j)\right\}$ are the polynomials orthonormal on the discrete uniform distribution on $K$ points given in section 2. Define $G$ by

$$
\sqrt{ } K G=\left(\begin{array}{cccc}
g_{1}(1) & \cdots & g_{K-1}(1) & 1 \\
\vdots & \vdots & \vdots & \vdots \\
g_{1}(K) & \cdots & g_{K-1}(K) & 1
\end{array}\right)
$$

and put $H=G \otimes G$. In defining $U_{\ell m}$ as in section 2, note that for the Andersontype tables we are considering, $\sum_{i=1}^{K} \sum_{j=1}^{K} N_{i j}=n K$ and so for $\ell=1, \cdots, K$ and $m=1, \cdots, K$, put

$$
U_{\ell m}=\sum_{i=1}^{K} \sum_{j=1}^{K} N_{i j} g_{\ell}(i) g_{m}(j) / \sqrt{(n K)}
$$

We seek to express $Y$ and hence $(K-1) A / K$ in terms of the $U_{\ell m}$. First note that

$$
U_{\ell m}=\sum_{i=1}^{K} \sum_{j=1}^{K}\left(N_{i j}-\frac{n}{K}\right) g_{\ell}(i) g_{m}(j) / \sqrt{(n K)} .
$$

because $\sum_{i=1}^{K} g_{\ell}(i)=\sum_{j=1}^{K} g_{m}(j)=0$ from the orthogonality. Second note that for $m=1, \cdots, K$

$$
U_{K m}=\sum_{i=1}^{K} \sum_{j=1}^{K}\left(N_{i j}-\frac{n}{K}\right) g_{m}(j) /(K \sqrt{(n)}=0
$$

as $\sum_{i=1}^{K}\left(N_{i j}-\frac{n}{K}\right)=0$. Similarly $U_{\ell K}=0$ for $\ell=1, \cdots, K$. Now note that

$$
K H^{T} X=\left(\sqrt{ } K G^{T} \otimes \sqrt{ } K G^{T}\right) X
$$


is a $K^{2}$ by 1 vector with typical element

$$
\sum_{i=1}^{K} \sum_{j=1}^{K} X_{i j} g_{\ell}(i) g_{m}(j)=U_{\ell m} K \sqrt{\frac{K}{K-1}}
$$

Thus $Y=[(K-1) / K] H^{T} X$ has typical element $U_{\ell m} \sqrt{\frac{K-1}{K}}$. Since we noted previously that the elements of $Y$ are asymptotically mutually independent and asymptotically standard normal, it follows that this is also true for the $U_{\ell m} \sqrt{\frac{K-1}{K}}$.

\section{The First Component: Page's Statistic}

We now identify $U_{11} \sqrt{\frac{K-1}{K}}$. Note that $g_{1}(j)=a j+b, j=1, \cdots, K$, in which

$$
\begin{aligned}
& a=\sqrt{ }\left\{12 /\left(K^{2}-1\right)\right\} \text { and } \\
& b=-\sqrt{ }\{3(K+1) /(K-1)\}=-\{(K+1) / 2\} a=-a \mu \text { if } \mu=(K+1) / 2 .
\end{aligned}
$$

The rank sum for treatment $i, R_{i}$, is $\sum_{j=1}^{K} j N_{i j}, i=1, \cdots, K$. The Page test statistic is

$$
L=\sum_{i=1}^{K} i R_{i}=\sum_{i=1}^{K} \sum_{j=1}^{K} i j N_{i j}
$$

Recall that

$$
\mu_{L}=n K(K+1)^{2} / 4 \text { and } \sigma_{L}^{2}=n K^{2}(K-1)(K+1)^{2} / 144
$$

Now

$$
\begin{aligned}
U_{11} \sqrt{\frac{K-1}{K}} & =\sqrt{\frac{K-1}{K}} \sum_{i=1}^{K} \sum_{j=1}^{K} N_{i j} g_{1}(i) g_{1}(j) / \sqrt{(n K)} \\
& =a^{2} \sqrt{\frac{K-1}{K}} \sum_{i=1}^{K} \sum_{j=1}^{K} N_{i j}(i-\mu)(j-\mu) / \sqrt{(n K)} \\
& =\frac{12}{K^{2}-1} \sqrt{\frac{K-1}{n K^{2}}}\left\{\sum_{i=1}^{K} \sum_{j=1}^{K} i j N_{i j}-n K \mu^{2}\right\} \\
& =\frac{L-n K\left(\frac{K+1}{2}\right)^{2}}{\sqrt{\frac{n(K-1) K^{2}(K+1)^{2}}{144}}}=Z_{L},
\end{aligned}
$$

as in section 4 . 


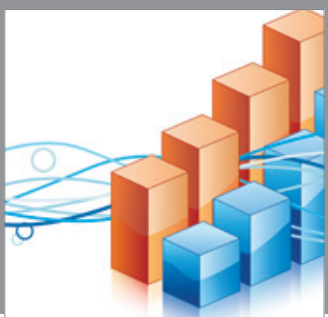

Advances in

Operations Research

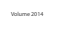

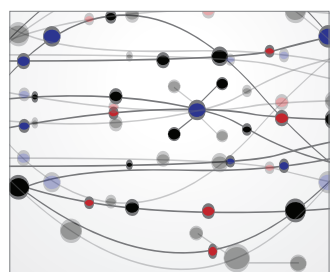

\section{The Scientific} World Journal
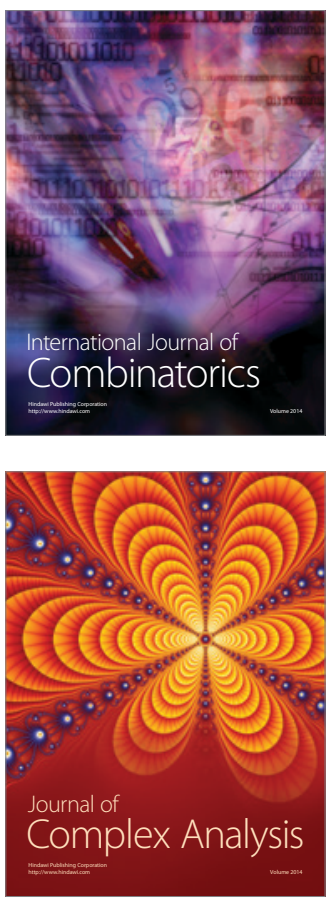

International Journal of

Mathematics and

Mathematical

Sciences
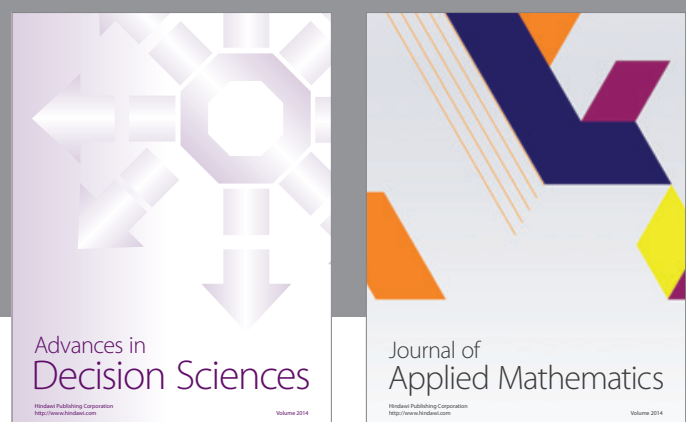

Journal of

Applied Mathematics
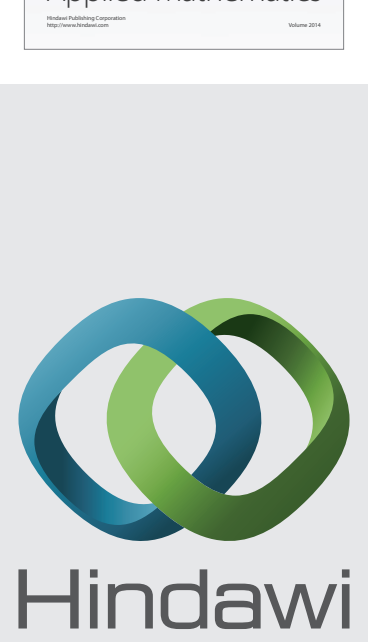

Submit your manuscripts at http://www.hindawi.com
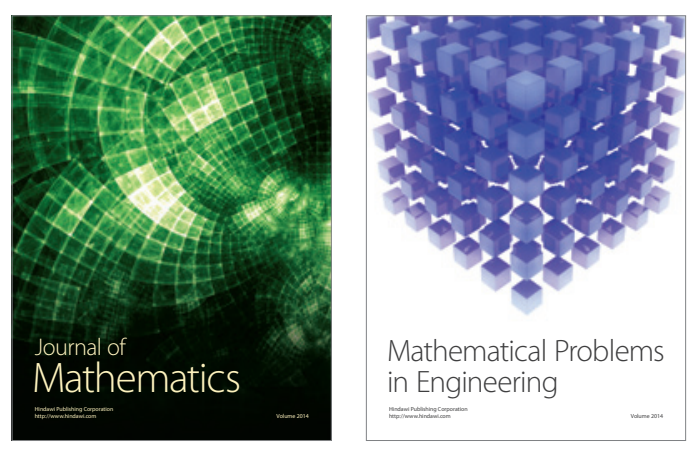

Mathematical Problems in Engineering
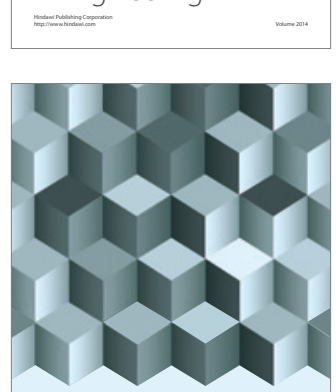

Journal of

Function Spaces
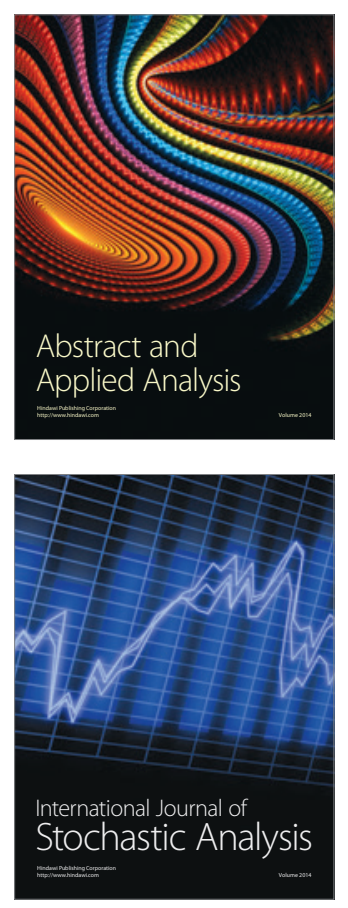

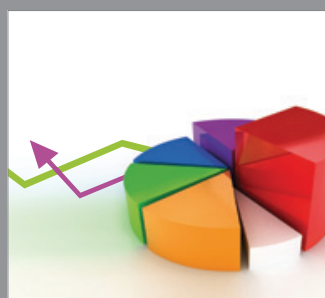

ournal of

Probability and Statistics

Promensencen
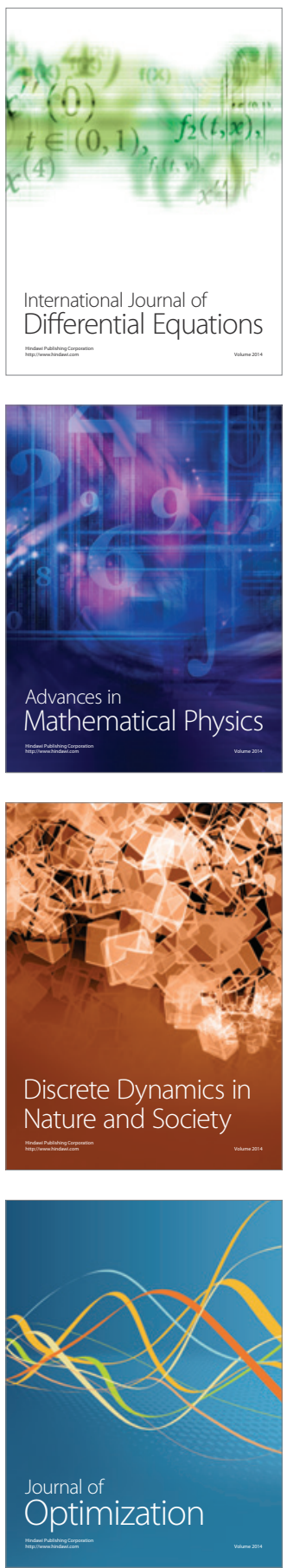